\title{
ESTÉTICA EN ODONTOPEDIATRÍA: RESTAURACIÓN INDIRECTA CON RESINA COMPUESTA EN DENTICIÓN PRIMARIA
}

Aesthetics in Pediatric Dentistry: Indirect restoration with composite resin in primary dentistry

Fecha de Recepción: 12 de agosto 2019
Estética em Odontopediatria: Restauração indireta com resina composta na dentição decídua

Aceptado para su publicación: 01 de octubre 2019

\section{Autores: \\ María L. Elizondo ${ }^{1}$ \\ María M. Medina \\ Sandra Martínez ${ }^{2}$ \\ Roxana E. Ojeda ${ }^{1}$ \\ Andrea V. Galiana ${ }^{1}$}

1. Área Disciplinar Odontopediatría. Módulo Atención Integral del Niño y Adolescente. Facultad de Odontología. Universidad Nacional del Nordeste. Argentina.

2. Área Disciplinar Práctica Clínica Preventiva I. Módulo Introducción a la Práctica Preventiva. Módulo Introducción a la Práctica Clínica. Facultad de Odontología. Universidad Nacional del Nordeste. Argentina.

Correspondencia: María L. Elizondo

Correo electrónico: marialidiaelizondo@yahoo.com.ar mlelizondo@odn.unne.edu.ar

Conflicto de intereses: los autores declaran no tener conflictos de interés.

Fuente de financiamiento: autofinanciado.

\section{Resumen}

El objetivo del presente trabajo fue realizar una restauración indirecta de resina de fotocurado de clase II en un segundo molar primario y presentar los pasos operatorios realizados como una alternativa estética terapéutica. Paciente de 8 años de edad de sexo femenino asistió para su rehabilitación integral a la Especialidad de Odontopediatría de la Facultad de Odontología de la UNNE. Al examen clínico la pieza dentaria 6.5 presentó una caries inactiva de Clase II compleja, en la cual dentro del plan de tratamiento rehabilitador se decidió realizar una restauración indirecta de resina de fotocurado. Las restauraciones indirectas para molares con resina de fotocurado confeccionado extraoralmente, son una posible alternativa estética, permitiendo optimizar las condiciones clínicas de trabajo y mejorar las propiedades finales del material restaurador.

Palabras clave: molares primarios, resina compuesta, incrustación, fotocurado.

\begin{abstract}
The aim of this work was to perform an indirect restoration using light curing resin in a second primary molar and present the operative steps performed as a therapeutic aesthetic alternative. 8-year-old female patient went to the Pediatric Dentistry Specialty office in the Dental School of the UNNE for her integral rehabilitation. The clinical examination showed a second primary right molar that presented a complex class II inactive caries. It was decided to perform an indirect restoration with photocuring resin as part of the rehabilitation treatment plan. Indirect restorations for molars with photocured resin made extraorally are a possible aesthetic alternative, making it possible to op-
\end{abstract}




\section{REFO}

VOL. XIII | N 1| AÑO 2020

ISSN 1668-7280

ISSN-E 2683-7986 timize the clinical conditions of work and improve the final properties of the restorative material.

Key words: primary molars, composite resin, inlay, light-cured.

\section{Resumo}

O objetivo do presente trabalho foi realizar uma restauração indireta da resina de cura classe II em um segundo molar primário e apresentar as etapas operatórias realizadas como alternativa estética terapêutica. Paciente do sexo feminino, 8 anos de idade, atendida na Especialização em Odontopediatria da Faculdade de Odontologia da UNNE pela sua reabilitação integral. Ao exame clínico, a peça dental 6,5 apresentou uma complexa cárie inativa classe II, na qual, dentro do plano de tratamento de reabilitação, optou-se pela restauração indireta da resina de cura. Restaurações indiretas para molares com resina curada extra-oralmente são uma alternativa estética possível, permitindo otimizar as condições clínicas de trabalho e melhorar as propriedades finais do material restaurador.

Palavras-chave: molares primários, resina composta, incrustação, fotopolimerização.

\section{Introducción}

Las lesiones de Clase II muy frecuentes en Odontopediatría, han sido siempre muy difíciles de restaurar. Ante el avance de la caries dental, por sus delgados espesores dentarios y grandes prominencias de cámaras y cuernos, se producen rápidas exposiciones pulpares.

Los niños con defectos dentales del desarrollo, destrucciones coronarias extensas producto de la caries dental o traumatismos, presentan como consecuencia cavidades complejas, planteando un desafío en la planificación del tratamiento restaurador del paciente pediátrico. Las dificultades inherentes a la técnica restauradora empleada y a la colaboración del niño, son factores de importancia al momento de decidir el tipo de restauración a realizar ya que requieren largos tiempos operatorios ${ }^{1,2}$.
El Odontopediatra ante estas situaciones, prioriza detener el avance de la caries y restaurar adecuadamente, consiguiendo un buen sellado marginal. Hoy en día, continúan evaluando el material más adecuado a utilizar para permitir la recuperación anatomo funcional de la pieza dentaria primaria. Una posible solución estética, acompaña la toma de decisión de realizar o no el tratamiento sugerido y generalmente solicitado por los padres.

Fukuyama et al. ${ }^{3}$, observaron que en 533 niños entre 1 y 13 años, el tipo de restauración más frecuentemente utilizado en el sector posterior en dientes primarios era la corona de acero inoxidable y las incrustaciones metálicas. En la actualidad, el uso de coronas metálicas en niños muy pequeños entre 18 y 36 meses, sigue siendo un aliado en caries de aparición temprana, pero los cuestionamientos y exigencias estéticas por parte de los padres fueron creciendo $^{4-6}$. En 1997, Boj et al.7, ya preconizaban la nueva tendencia de restauraciones indirectas con resinas en el sector posterior.

Las restauraciones estéticas directas en niños en el sector posterior, son complicadas de realizar, por ello se considera a la resina indirecta de fotocurado $^{8}$, una restauración alternativa en preparaciones de cavidades Clase II en dientes primarios, ya que habitualmente la corona metálica, de acuerdo a la profundidad de la lesión, terminaba siendo la restauración de elección para garantizar la longevidad de la pieza dentaria primaria.

Actualmente, las nuevas tecnologías diseño asistido por computadora y tecnología de fabricación asistida por computadora (CAD/ CAM, por sus siglas en inglés) se utilizan para tratar caries prolongadas y/o profundas en molares primarios, permite también facilitar las restauraciones estéticas en dentición primaria?.

El objetivo del presente trabajo fue realizar una restauración indirecta de resina de fotocurado de clase II en un segundo molar primario y presentar los pasos operatorios realizados como una alternativa estética terapéutica. 


\section{5}

CASO CLÍNICO
Revista de la Facultad de Odontología

Estética en Odontopediatría: restauración indirecta con resina compuesta en dentición primaria

\section{REFO}

VOL. XIII | N 1| AÑO 2020

ISSN 1668-7280

ISSN-E 2683-7986

\section{Caso clínico}

Paciente de 8 años de edad de género femenino, asistió junto a su padre para una consulta por caries dental a la Especialidad de Odontopediatría de la Facultad de Odontología de la UNNE para su rehabilitación odontológica integral.

Al examen clínico y radiográfico la pieza dentaria 6.5 (Figura 1) presentaba una caries inactiva no penetrante de Clase II compleja, por lo que se decidió realizar dentro de una planificación como parte del plan de tratamiento rehabilitador una restauración indirecta con resina de fotocurado.
Una vez eliminado el tejido cariado se dejó la dentina oscura remineralizada. La preparación cavitaria se realizó utilizando una fresa troncocónica de extremo redondeado permitiendo una ligera divergencia hacia oclusal de las paredes vestibular, palatina y mesial. La unión de las paredes con el piso mediante el uso de esta fresa permitió que los ángulos internos de la preparación resulten redondeados para evitar áreas de tensión interna. Los márgenes de la preparación no se biselaron para evitar espesores delgados del material de restauración que faciliten su fractura. La evidencia científica ${ }^{10}$, recomienda dejar un escalón proximal o dar una ligera pendiente del piso de la preparación hacia proximal (Figura 2).

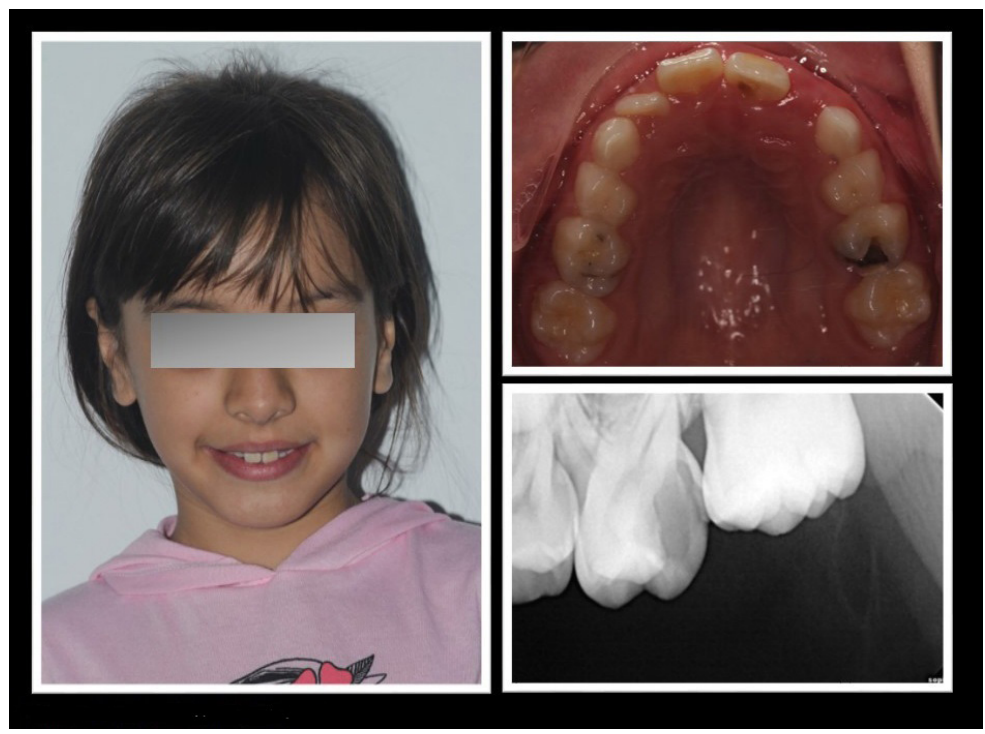

Figura 1. Evaluación clínica y radiográfica.

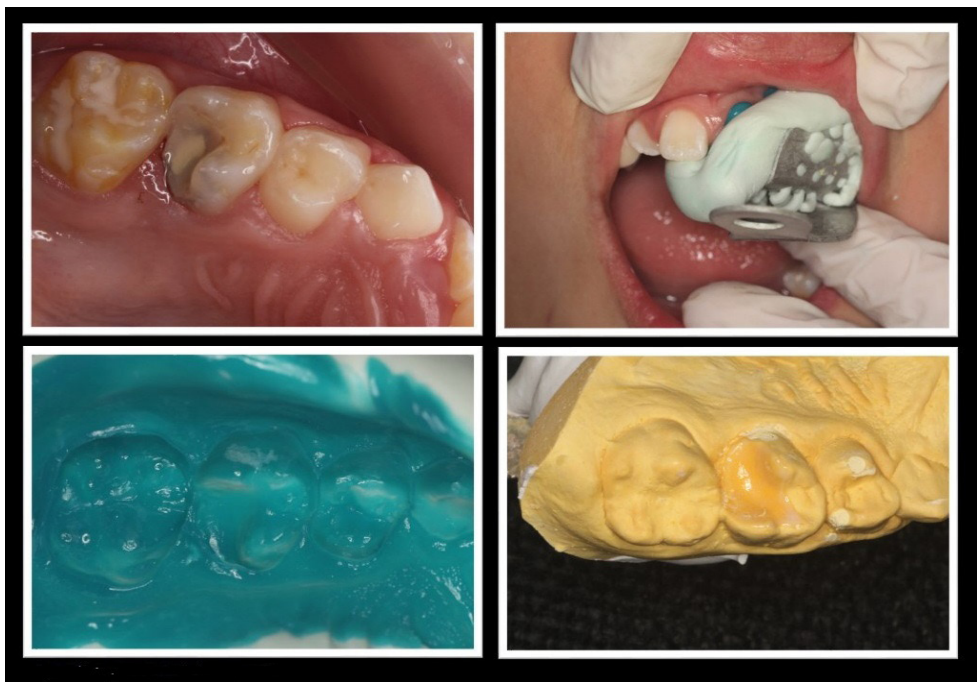

Figura 2. Preparación cavitaria e impresión. 
Una vez realizada la preparación cavitaria, se lavó y secó con jeringa triple, colocando un liner de Hidróxido de Calcio fraguable en la zona más profunda del piso cavitario de la preparación. A fin de regularizar algunas zonas de retención, se colocó una Resina fluida híbrida de fotocurado Master Flow (Biodinámica ${ }^{\circledR}$ ). Posteriormente se tomó una impresión parcial con silicona por condensación utilizando la técnica de doble pasta en dos pasos (Coltène/Whaledent ${ }^{\circledR}$ ) aportando una gran elasticidad, estabilidad dimensional y precisión. Se realizó el vaciado con yeso amarillo tipo IV (Whip Mix Silky Rock $($ ) y se montó en un oclusor con su antagonista para el control de la oclusión durante la preparación de la incrustación. Se colocó en primer lugar una laca transparente espaciadora sobre el modelo a fin de evitar la adhesión de la resina al yeso (Figura 2). Con la técnica incremental, se colocó la resina Brilliant NG (Coltène $\left.{ }^{\circledR}\right)$ de color A2 realizando el fotopolimerizado de cada capa durante 60 segundos hasta finalizar la preparación (Figura 3), siguiendo pautas de la literatura internacional ${ }^{7-10}$.

Con la ayuda de un bisturí se retiró la restauración del modelo de yeso y se realizó el lavaje con solución fisiológica para eliminar residuos de polvo. Previo al cementado, se trató la restauración empleando arena con óxido de aluminio grano 80 um durante 15 segundos y ácido fosfórico al 35\% durante 30 segundos, se lavó y secó, según pautas preestablecidas ${ }^{11}$.

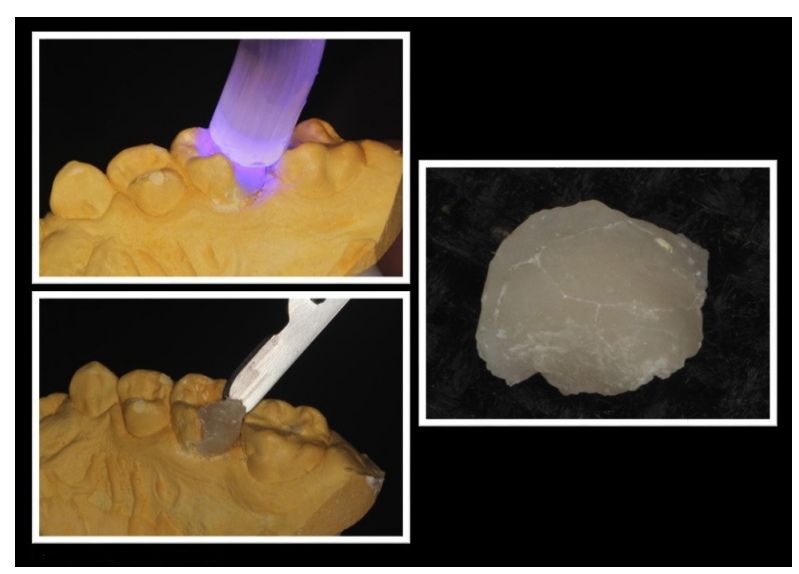

Figura 3. Confección de la restauración.
Se controló la adaptación de la incrustación a la preparación cavitaria, (Figura 4) ingresando en la cavidad sin necesidad de hacer retoques internos. Para el tratamiento de la cavidad se realizó el grabado ácido al $35 \%$ durante 30 segundos. Posteriormente, se colocó adhesivo OptiBond ${ }^{\mathrm{TM}}(\mathrm{KERR} \circledast)$ a la incrustación y a la cavidad sin polimerizar a fin de evitar un espesor entre ambas, luego se cementó con una resina de curado dual (Figura 4). Se utilizó este material de fijación por presentar las siguientes ventajas: tiempo controlado de cementado, estética, baja microfiltración y mejores propiedades físicas ${ }^{12,13}$. Finalmente, se realizó el control de la oclusión y el pulido correspondiente, vista de la Incrustación de resina finalizada, vista vestibular y oclusal (Figura 5). Siguiendo pautas internacionales,

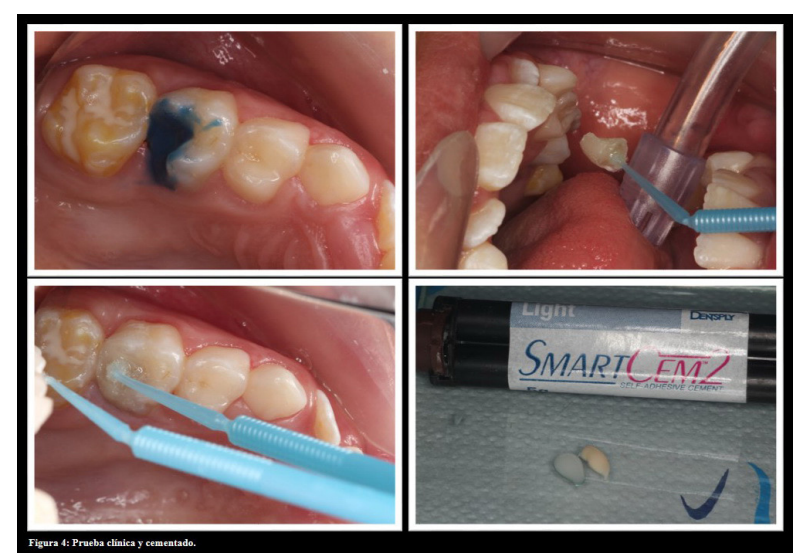

Figura 4. Prueba clínica y cementado.

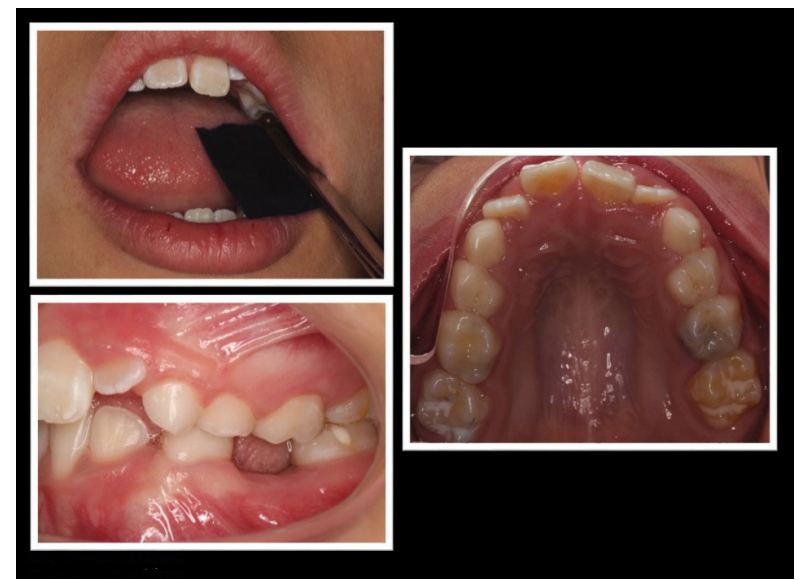

Figura 5. Control de la oclusión 


\section{REFO}

VOL. XIII | N 1| AÑO 2020

ISSN 1668-7280

ISSN-E 2683-7986 se realizó un control clínico a los 6 meses $^{14}$, observándose buena adaptación en el margen restaurador, ausencia de sintomatología dolorosa y tejido gingival sano (Figura 6).

\section{Discusión}

En la consulta diaria, es común observar lesiones de Clase II complejas en dentición primaria. Esta situación nos lleva a plantearnos el tipo de restauración adecuado considerando la satisfacción del niño 14 y del padre en cuanto a la estética final, tratando de realizar la mínima intervención y eliminación de tejido dentario posible. Teniendo presente además el grado de colaboración y comportamiento del niño ${ }^{15}$.

La técnica de restauración indirecta en niños, presenta la ventaja de disminuir los tiempos operatorios clínicos, permite reducir la ansiedad del paciente y realizar la restauración fuera de la boca pudiendo controlar los factores de contracción de polimerización, estética y adaptación funcional que generan una restauración confiable ${ }^{10}$.

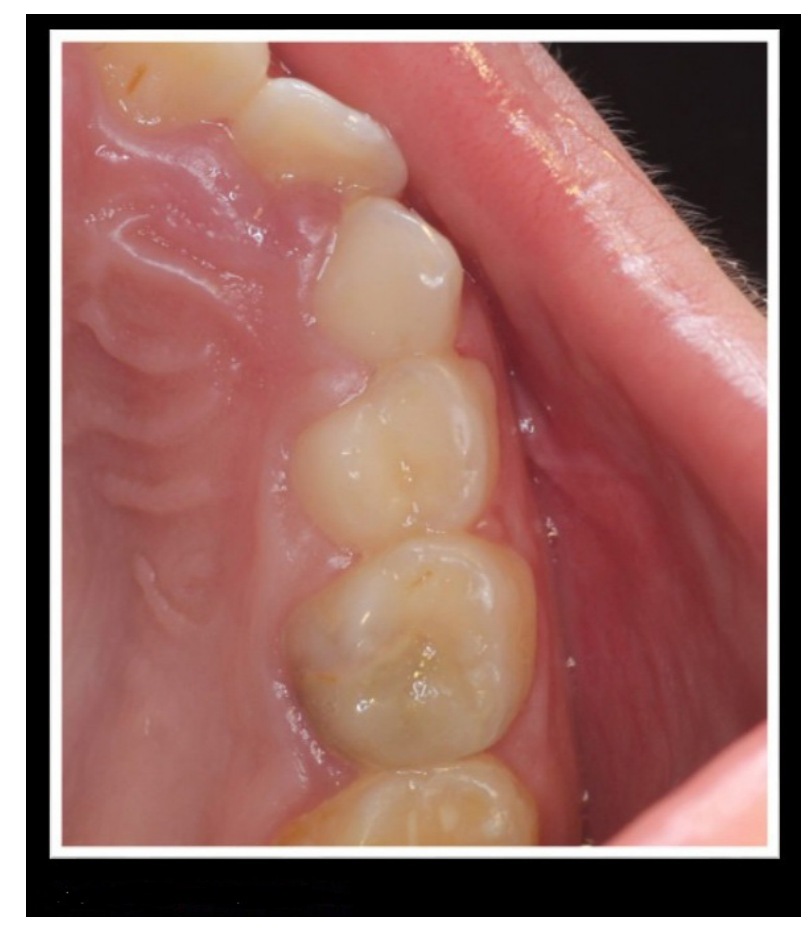

Figura 6. Control clínico a los 6 meses.
Fukuyama et al. ${ }^{3}$, hallaron mayor frecuencia de uso de incrustaciones metálicas en niños entre 5 y 9 años, en el maxilar superior en 1 y 2 molares primarios, y confirman que continúa esta tendencia hoy en día. Bertoldy ${ }^{16}$, afirma que las incrustaciones con resinas compuestas cumplen funciones similares a las incrustaciones metálicas y cerámicas en lo que se refiere a la rehabilitación de piezas dentales posteriores con gran daño estructural. Ambos autores coinciden en que el éxito obtenido en la adaptación de la incrustación, se atribuye a una adecuada toma de impresiones y a la delimitación y/o confección de la incrustación.

Con el gran avance de las resinas, las incrustaciones estéticas se consideran una buena opción para el tratamiento de cavidades amplias ${ }^{17-19}$.

La adhesión a esmalte y dentina en Odontopediatría, ha mejorado considerablemente en los últimos años. Sin embargo, es importante tener en cuenta las diferencias en la composición del esmalte y dentina en ambas denticiones, al momento de realizar el grabado ácido tanto en esmalte como en dentina ${ }^{16,17}$.

Al secado, el esmalte debe observarse con un aspecto blanco tiza y la dentina sin resecar, aplicándose un sistema adhesivo que combine la imprimación y adhesión. La técnica debe ser cuidadosamente realizada de acuerdo a las instrucciones del fabricante, para mejorar la retención, minimizar la microfiltración y reducir la sensibilidad18.

Las restauraciones indirectas con resina compuesta de fotocurado confeccionada extraoralmente, constituyen una alternativa estética de restauración en molares primarios. Si bien se requiere de la colaboración del paciente para su preparación, esta técnica posibilita optimizar las condiciones clínicas de trabajo y mejorar las propiedades finales de la restauración.

\section{Referencias Bibliográficas}

1. Fernández MR, Fontés ST, Carvalho RV, Simancas Y, ViIlalón M. Restauración semidirecta de resina compuesta: una alternativa para dientes posteriores - reporte de caso clínico. Acta Odontol Venezol [Internet]. 2010 [citado 
14 Feb 2019];48 (3):1-14. Disponible en: https://www. actaodontologica.com/ediciones/2010/3/art-14/

2. Mittal HC, Goyal A, Gauba K, Kapur A. Clinical Performance of Indirect Composite Onlays as Esthetic Alternative to Stainless Steel Crowns for Rehabilitation of a Large Carious Primary Molar. J Clin Pediatr Dent. 2016;40(5):345-52. doi: 10.17796/1053-4628-40.5.345 3. Fukuyama T, Oda S, Yamashita H, Sekiguchi H, Yakushiji M. Clinical survey on type of restoration in deciduous teeth. Bull Tokyo Dent Coll. 2008;49(1):41-50. doi: 10.2209/ tdcpublication.49.41

4. Ramanathan G, White GE. A direct composite onlay technique for primary molars using a light/heat curing system. J Clin Pediat Dent. 1990;15(1):13-16. PMID: 2098080

5. Geurtesen W, Hullmann U, Gockel H. Restauraciones inlay/onlay de composite elaboradas indirectamente (I). Quintaessence (Ed. Esp) 1989;2(9):203-205.

6. Akhlaghi N, Hajiahmadi M, Golbidi M. Attitudes of Parents and Children toward Primary Molars Restoration with Stainless Steel Crown. Contemp Clin Dent . 2017; 8(3):421-426. doi: 10.4103/ccd.ccd_379_17

7. Gatón P, Espasa E, Boj JR. Restauraciones fototermocuradas en dentición temporal. Anales de Odontoestomatología [Internet]. 1997 [citado 14 Feb 2019]; 2:70-4. Disponible en: http://diposit.ub.edu/dspace/bitstream/2445/21790/1/120467.pdf

8. Leinfelder KF. Indirect posterior composite resins. Compend Contin Educ Dent. 2005;26(7):495-503. doi: 10.14219/jada.archive.1988.0039

9. Dursun E, Monnier-Da Costa A, Moussally C. Encimeras compuestas de CAD / CAM para la restauración de molares primarias. J Clin Pediatr Dent. 2018;42(5):349354. doi: 10.17796/1053-4625-42.5.5

10. Cedillo Valencia JJ, Cedillo Félix JE. Restauraciones indirectas de resina en una sola visita. Reporte de un caso clínico. ADM [Internet]. 2013 [citado 14 Feb 2019];70(6):329-338. Disponible en: https://www.medigraphic.com/pdfs/adm/od-2013/od136i.pdf
11. Hopp CD, Land MF. Considerations for ceramic inlays in posterior teeth: a review. Clin Cosmet Investig Dent. 2013;5:21-32. doi: 10.2147/CCIDE.S42016

12. Coban BG, Kirzioglu Z, Altun AC. Can onlay's be an alternative restoration for severely damaged primary teeth. Eur J Dent. 2017;11(1):48-52. doi: 10.4103/13057456.202616

13. Cantoro A, Goracci C, Papacchini F, Mazzitelli C, Fadda GM, Ferrari M. Effect of pre-cure temperature on the bonding potential. J Den Mat. 2008;24(5):577-583. doi: 10.1016/j.dental.2007.06.012

14. Mittal N, Srivastava B. Clinical outcomes of indirect composite restorations for grossly mutilated primary molars: a clinical observation. Gen Dent. 2015; 63(3):16-21. doi: 10.5005/jp-journals-10005-1255

15. Basso ML. Tratamiento de la caries dentinaria en Odontopediatría. Remoción parcial de la dentina cariada. Rev Asoc Odontol Argent. 2010;98(2):133-44. ID: lil594971

16. Bertoldy A. Incrustaciones de resinas compuestas. Rev Asoc Odontol Argent. 2004;92(3):253-64. ID: lil-384887 17. Garcia-Godoy F, Donly KJ. Dentin/enamel adhesives in pediatric dentistry. Pediatr Dent. 2002;24(5):462-64. PMID: 25905654

18. Kirzioglu Z, Ceyhan D, Sengul F, Altun AC. Three-dimensional finite element analysis of the composite and compomer onlays in primary molars. Comput Methods Biomech Biomed Engin. 2019;22(10):936-941. doi: 10.1080/10255842.2019.1604951

19. Wada K, Miyashin M. New techniques for producing aesthetic, direct full-crown composite resin restorations for primary molars: a 24-month follow-up study of eight cases. Eur J Paediatr Dent. 2015;16(3):205-9. PMID: 26418923 